\title{
Nutritional Interventions for the Prevention of Cognitive Impairment and Dementia in Developing Economies of East Asia: Systematic Review and Meta-analysis
}

\author{
Andrea McGrattan $^{1}$, Carla van Aller ${ }^{1}$, Alla Narytnyk ${ }^{2}$, Daniel Reidpath ${ }^{3}$, Pascale Allotey ${ }^{3}$, \\ Devi Mohan $^{3+}$, Blossom Stephan ${ }^{1}$, Louise Robinson ${ }^{1}$ and Mario Siervo ${ }^{4}$ \\ ${ }^{1}$ Institute of Health and Society, and Institute of Ageing; Newcastle University, Newcastle, United Kingdom, \\ ${ }^{2}$ Institute of Genetic Medicine, Newcastle University, Newcastle, United Kingdom, \\ ${ }^{3}$ Jeffrey Cheah School of Medicine and Health Sciences, Monash University Malaysia, Selangor, Malaysia and \\ ${ }^{4}$ School of Life Sciences, The University of Nottingham Medical School, Nottingham, United Kingdom
}

\begin{abstract}
Introduction: Dementia represents one of the impending global health challenges, and low and middle-income countries (LMICs) are projected to greatly contribute to the rising dementia global burden. Currently, there is a lack of pharmacological treatment for dementia and therefore research efforts have focused on prevention, with the identification of early lifestyle, demographic and nutritional risk factors. In particular, diet may be an important modifiable risk factor for maintenance of cognitive health in later life. There are plausible suggestions to support the synergistic effects of certain nutrients, such as polyphenols, unsaturated fats and antioxidant vitamins, in having a beneficial role in the modulation of oxidative stress and neuro-inflammation - processes associated with cognitive decline. Therefore, the aim of this systematic review was to evaluate the current evidence on nutritional interventions for the prevention of dementia in developing economies in East Asia.
\end{abstract}

Materials and Methods: Four comprehensive medical databases were searched from inception until February 2019: MEDLINE, EMBASE, PsycINFO and Scopus. The literature search was restricted to randomised clinical trials [RCTs], conducted in adult humans [ $\geq 18$ years], assessing the effect of nutritional interventions on cognitive performance, and / or incidence of mild cognitive impairment [MCI] or dementia. The outcome of interest for the meta-analysis was: [1] global cognitive performance and [2] domain specific cognitive performance. Data was pooled by random model analysis and estimates of effect size were given for each domain and sub-categorised according to the type of nutritional intervention.

Results: Twenty-two RCTs were included, of which, sixteen studies showed significant beneficial effects in favor of the nutritional intervention based on single neuropsychological test scores and / or scores of global cognitive assessment tools. Sixteen studies had sufficient data reported for meta-analysis, and marginally significant beneficial effects were found on global cognitive performance in elderly for micro-nutrient supplementation $[\mathrm{n}=4$ studies, $\mathrm{n}=451$ participants, std mean difference: 0.41 [-0.03; 0.84], $\mathrm{p}=$ 0.07], and EPA / DHA supplementation [ $\mathrm{n}=4$ studies, $\mathrm{n}=373$ participants, std mean difference $0.57[-0.01 ; 1.14], \mathrm{p}=0.06]$.

Discussion: Several promising strategies, such as B-vitamin supplementation, EPA / DHA supplementation and nutrition and lifestyle counselling interventions, seem to be able to decrease age-related cognitive decline in East Asia. Large, good quality, long term trials are needed to confirm these findings, to further evaluate the role of nutritional interventions on cognitive function and to identify if these interventions are feasible and effective to decrease dementia incidence in developing economies, like East Asia.

\section{Conflict of Interest}

There is no conflict of interest

\#The original version of this article was submitted with incorrect author information. A notice detailing this has been published and the error rectified in the online PDF and HTML copies. 